\title{
Remanent Magnetic Flux Distribution in Superconducting-Ferromagnetic Layered Heterostructures
}

\author{
A Baskys $^{1}$ (D) $\cdot$ A Patel $^{1}$ - V. Climente-Alarcon ${ }^{1} \cdot$ B A Glowacki $^{1,2,3,4}$
}

Received: 14 May 2018 / Accepted: 16 January 2019 / Published online: 22 February 2019

(C) The Author(s) 2019

\begin{abstract}
Stacks of superconducting tape, made of second-generation coated conductors, present an interesting alternative to bulk superconductors. The layered geometry allows incorporation of other functional, namely ferromagnetic, materials. The ferromagnetic material changes the field configuration within the superconductor and can be used to shape or divert the flux density from its interior. This can be used to optimise the remanent flux density after magnetisation. FEM simulations are employed to test the performance of a variety of superconductor/ferromagnet structures and explain the role of the ferromagnetic material in the composite structure. Cases where trapped field is much higher and lower than remanent magnetisation of the ferromagnetic material are considered, showing that performance can be enhanced or maintained by using ferromagnetic material while reducing the usage of costly superconducting tape.
\end{abstract}

Keywords Trapped field magnet $\cdot$ HTS tape $\cdot$ Stack of tapes $\cdot$ HTS modeling

\section{Introduction}

A number of works have demonstrated that the use of ferromagnetic materials can improve the properties of trapped field magnets (TFMs). Most of the work so far has been focused on trapped field magnets made of bulk superconductors (SCs), where ferromagnetic materials (FMs) have shown the ability to tailor the magnetic field profile [1], reduce crossed-field demagnetisation [2] or just increase the trapped field above a bulk superconductor [3]. Other work on second-generation high-temperature superconducting (2G HTS) tapes using numerical modelling showed that magnetic shielding of filaments by ferromagnetic material reduces AC losses in selffield conditions due to decoupling of the filaments and, at the

A Baskys

ab857@cam.ac.uk

1 Applied Superconductivity and Cryoscience Group, Department of Materials Science and Metallurgy, University of Cambridge, 27 Charles Babbage Road, Cambridge CB3 OFS, UK

2 Institute of Power Engineering, ul. Mory 8, 02-981 Warsaw, Poland

3 Department of Physics and Energy and Bernal Institute, University of Limerick, Castletroy, Limerick, Ireland

4 Epoch wires Ltd, Foxton, Cambridge CB22 6SA, UK same time, it increases the critical current of the composite [4]. Previous work in the group has shown that a composite TFM made of layers of HTS tape layered with ferromagnetic layers increased the trapped field above the stack as compared to a stack made of only HTS tape [5]. However, the magnetisation was performed using pulsed field magnetisation (PFM) where thermal effects have a significant role; hence, it is not clear if it was the thermal or magnetic properties of the ferromagnetic layers that have led to the improvement in trapped flux. This work aims to use finite element method (FEM) modelling to gauge the possible improvements to trapped field using ferromagnetic materials by simulating "fully saturated" TFM, similar to a condition after field cooling magnetisation (FCM). Several cases are modelled considering bulk FM material outside the stack or FM layered together with SC material with or without considering the anisotropy of the critical current $\mathrm{J}_{\mathrm{c}}(B, \theta)$.

\section{Methods and Techniques}

\subsection{Modelling Framework and Material Properties}

The FEM modelling was performed in Comsol Multiphysics 5.3a. An axis-symmetric model using $A$ formulation (magnetic vector potential) was set up to simulate a cylindrical stack. 

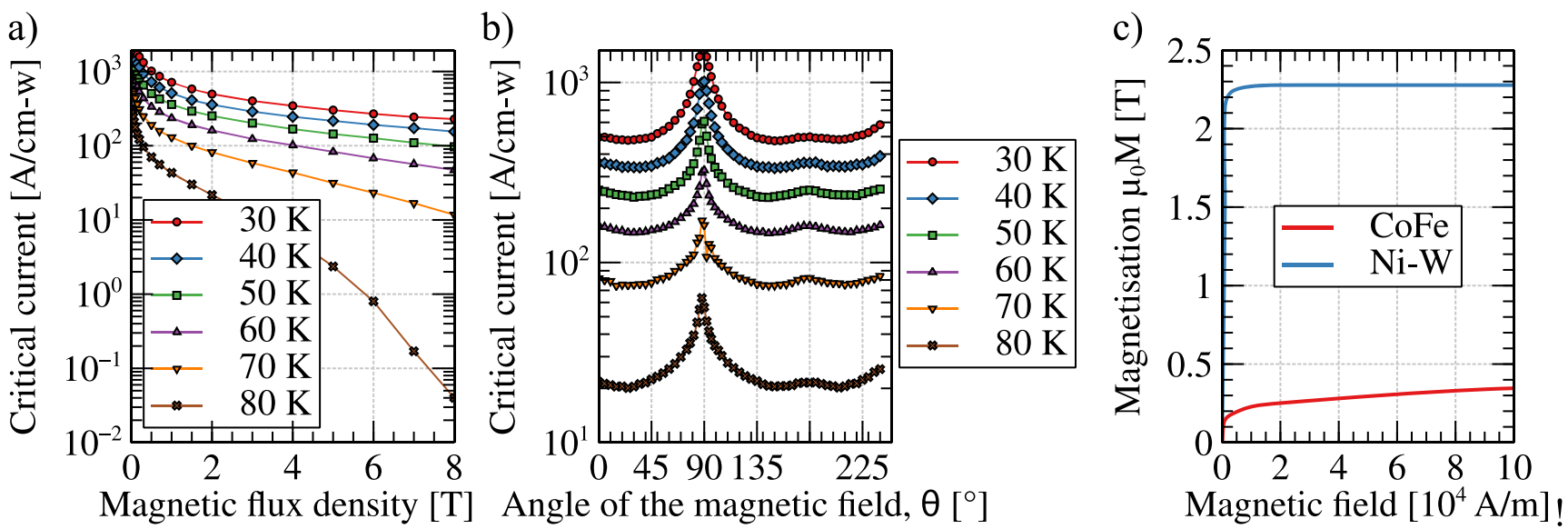

Fig. 1 Critical current data used for simulations for $B \| c$, i.e. $J_{\mathrm{c}}\left(B, T, \theta=0^{\circ}\right)$ is shown in a, while angular dependency of critical field $J_{\mathrm{c}}(B=2 \mathrm{~T}$, $T, \theta)$ is shown in $\mathbf{b}$. Magnetisation curves for magnetic materials used in the study (c)

A stationary (time-independent) study was used and the current density in the superconductor was set according to the $E-J$ power law:

$J=J_{\mathrm{c}}(B, \theta, T)\left(\frac{E}{E_{0}}\right)^{1 / n}$

where $J_{\mathrm{c}}(B, \theta, T)$ is the critical current density dependence on temperature, $T$, the magnetic field magnitude, $B$, and its direction, $\theta$, determined for a critical current criterion, $E_{0}$. While, $E$ is the average electric field in the stack after some relaxation period, in this case taken as $1 \mathrm{nV} / \mathrm{m}$ corresponding to relaxation of $\sim 5 \mathrm{~min}$ after magnetisation procedure, as determined by time-dependant simulations [6]. For this work, the $n$ value was fixed to 20 . The critical current data $J_{\mathrm{c}}(B, \theta, T)$ was taken from [7], corresponding to SuperOx superconducting tape and the data is shown in Fig. 1a, b.

A homogenised model was used, i.e. individual HTS layers were not modelled, instead the superconducting domains carried an engineering critical current density, $J_{\mathrm{e}}=I_{\mathrm{c}} / A$, where $A$ is the total cross-section area of the tape that corresponds to the SuperOx tape measured in [7].

a)

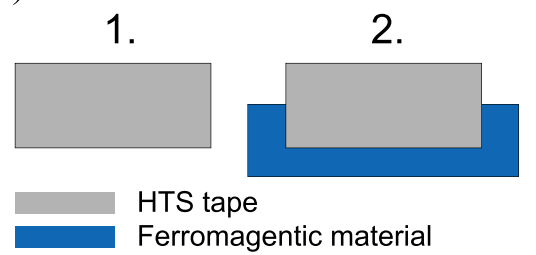

3.

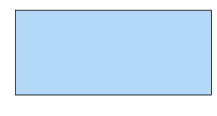

Fig. 2 The four cases considered in this work are shown in a, namely (1) stack with no modifications; (2) externally added ferromagnetic material; (3) intrinsic ferromagnetic material and (4) ferromagnetic material layered
Two ferromagnetic materials were considered in this work, namely Ni-W alloy, which is used for substrate in HTS tape made by RABiTS process (e.g. in American Superconductor tape) and a CoFe alloy, trademark Vacoflux 50, which has a very high saturation magnetisation close to $2.3 \mathrm{~T}$. Typical applications for Vacoflux 50 include rotating machinery. Magnetic properties for Ni-W can be found in [8], and data for Vacoflux was found in Comsol materials' library. The magnetisation curves are shown in Fig. 1c.

\subsection{Modelled Cases}

Four cases were considered, as depicted in Fig. 2a. In case 4, layers of both HTS and ferromagnetic material are $100 \mu \mathrm{m}$ thick, 120 layers in total. In each of the cases, the amount of $\mathrm{SC}$ material is the same; hence, stacks in cases $1-3$ are $6 \mathrm{~mm}$ thick, while in the case of 4-12 $\mathrm{mm}$.

In addition, the effect of ferromagnetic material was determined for several conditions: range of temperatures $(30-80 \mathrm{~K})$; anisotropic critical current $J_{\mathrm{c}}(B, T, \theta)$ vs $J_{\mathrm{c}}\left(B, T, \theta=0^{\circ}\right)$; and different width of tape, standard $12 \mathrm{~mm}$ and $46 \mathrm{~mm}$ wide (currently widest commercially available). Magnetic flux density and total flux $1 \mathrm{~mm}$

b)
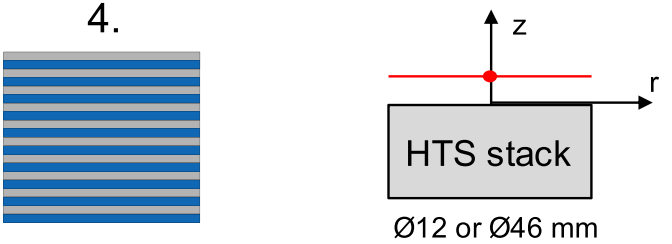

between HTS layers. The flux density is measured $1 \mathrm{~mm}$ above the centre stack surface while the magnetic flux is integrated over the surface above the stack at $1 \mathrm{~mm}$ height as shown in $\mathbf{b}$ 
a)

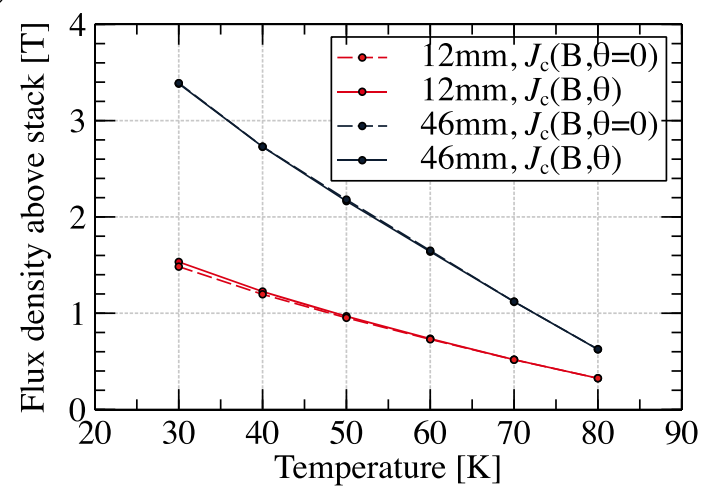

Fig. 3 Flux density $1 \mathrm{~mm}$ above the centre of the stack for 12- and 46mm-diameter stacks calculated using anisotropic critical current density $J_{\mathrm{c}}(B, \theta)$ and a case where orientational dependency of critical current

above the stack as shown in Fig. $2 \mathrm{~b}$ were taken as a merit index for comparison between different cases considered.

\section{Modelling Results}

\subsection{Stack with No Modifications}

As a comparison, a stack with no modifications or ferromagnetic materials was modelled, and the influence of anisotropic critical current density was determined. Figure 3a shows flux density above the stack for 12- and 46-mm-wide tape with and without considering anisotropy of critical current density. The figure shows that the anisotropy does not change the maximum flux density of the stack significantly, especially for 46mm-wide tape; however, Fig. $3 \mathrm{~b}$ shows that the flux density profile above the stack is changed; hence, the total flux above the stack will also be different. Figure $3 \mathrm{~b}$ shows that the flux density near the edge of the stack is enhanced, which corresponds to region of the stack where flux density within the stack is closer to $\theta=90^{\circ}$, corresponding to a peak in $J_{\mathrm{c}}(B, T, \theta)$. By considering the anisotropy, the enhancement in flux above the stack for $46-\mathrm{mm}$ tape ranges from $2 \%$ at $80 \mathrm{~K}$ to $10 \%$ at b)

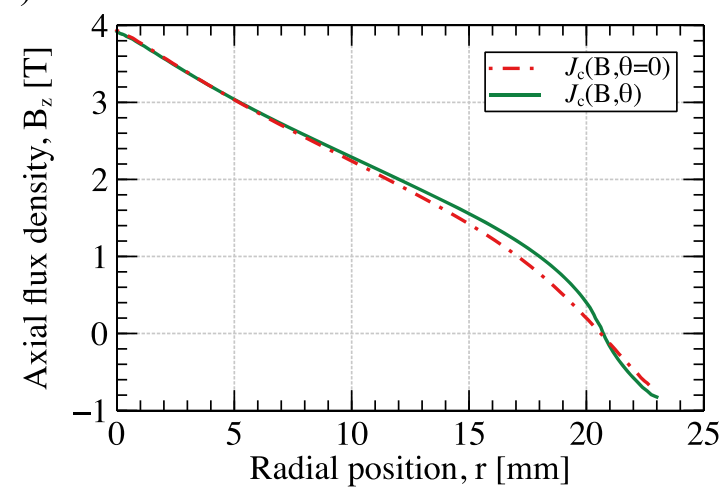

density is ignored $J_{\mathrm{c}}(B, \theta=0)(\mathbf{a})$. Magnetic flux density profile $1 \mathrm{~mm}$ above a $46-\mathrm{mm}$ stack at $30 \mathrm{~K}$ for the same two cases (b)

$30 \mathrm{~K}$. This shows that the anisotropic critical current density plays a more important role at low temperatures, which is consistent with $J_{\mathrm{c}}(B, T, \theta)$ curves in Fig. 1. The results obtained for the stack with no modifications will be used for comparison when ferromagnetic material is added.

\subsection{Stack with External Ferromagnetic Material}

An externally added ferromagnetic material can change field distribution inside and outside the HTS stack. A simple geometry depicted in Fig. 4a was used as a case study to determine optimal shape of the externally added ferromagnetic material, by changing dimensions $w_{1}, w_{2}, h_{1}$ and $h_{2}$. The optimisation was done using a Comsol optimisation module with constraint that $h_{2}<6 \mathrm{~mm}$ and $h_{1}, w_{1}<92 \mathrm{~mm}$, so that the top surface of the stack is clear as it would be for most applications and the width and thickness of the FM backplate no larger than twice the stack width-92 $\mathrm{mm}$ for 46-mm-wide tape.

It was found that the region of FM material, defined by $w_{2}, h_{2}$, does not contribute to increase the flux density above the stack; on the other hand, it was beneficial to continuously increase the $w_{1}$ and $h_{1}$. However, as shown in Fig. $5 \mathrm{~b}$ for 46-mm-diameter stack at $30 \mathrm{~K}$, increasing dimensions of the FM material beyond $h_{1}, w_{1}>50 \mathrm{~mm}$ yields very little a)

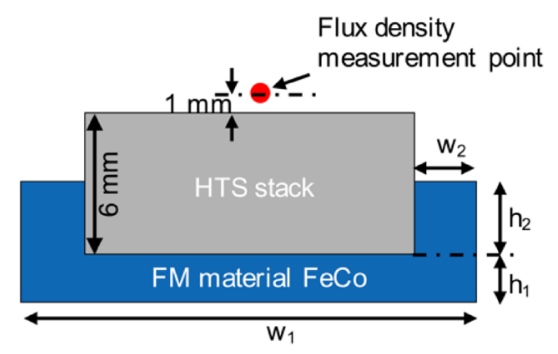

b)

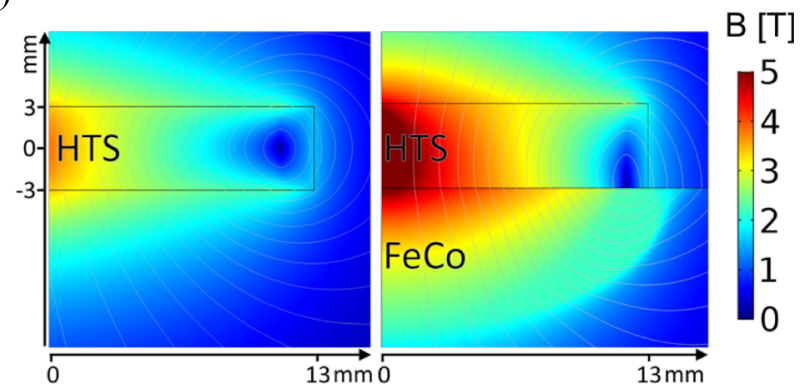

Fig. 4 Geometry of the HTS stack with external ferromagnetic material surrounding it (a) and flux density distribution around a stack on its own and with a large ferromagnetic slab underneath (b) (only half of the cross section is shown) 
a)

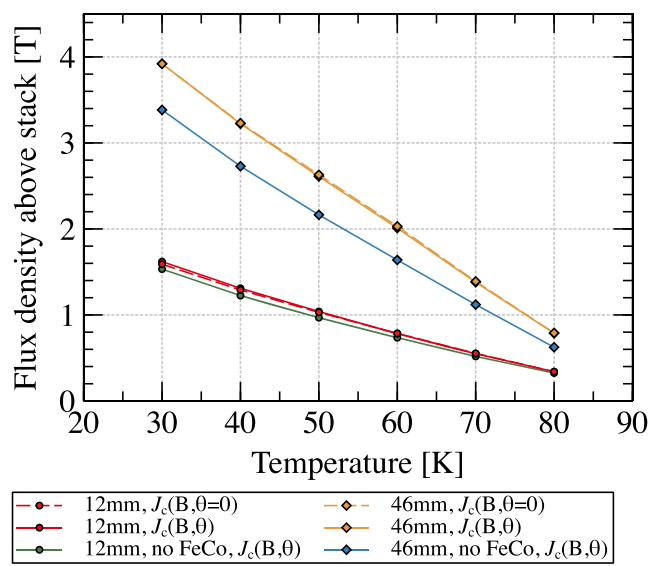

b)

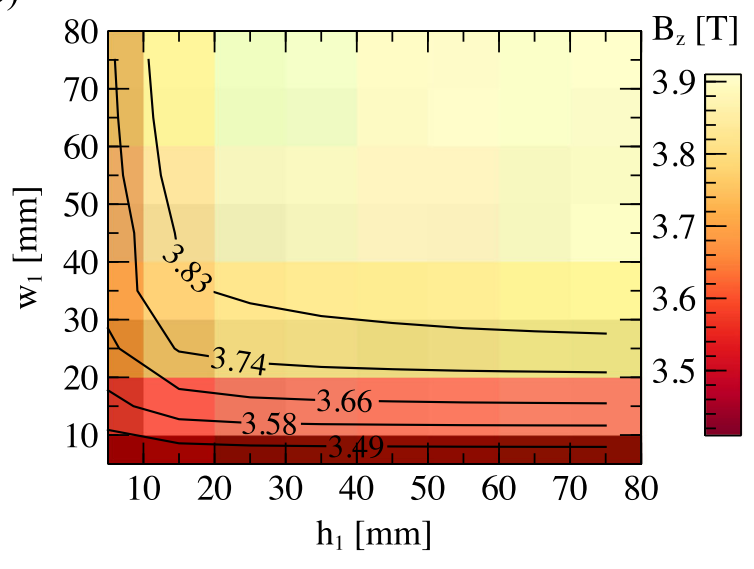

Fig. 5 Flux density above the stack with and without the ferromagnetic material (with optimised dimensions) for 12-and 46-mm-diameter stack with and without considering anisotropic $J_{\mathrm{c}}$ (a) and a plot of flux density above the 46-mm stack with various dimensions of the external FM material at $30 \mathrm{~K}$ (b)

improvement. The only exception where the optimal shape was different was the case of 46 -mm tape at $80 \mathrm{~K}$, where shape of the $J_{\mathrm{c}}(B, T, \theta)$ leads to the fact that the dimensions $w_{1}=15 \mathrm{~mm}, h_{1}=92 \mathrm{~mm}$ produced slightly higher field compared to $h_{1}=w_{1}=92 \mathrm{~mm}$ (maximum allowed dimensions in the study).Z

The improvements of using external FM material with optimised dimensions are detailed in Fig. 5. The results show that stacks made of wide tape benefit much more from the ferromagnetic material, and the improvement is close to $15 \%$ at $30 \mathrm{~K}$. While Fig. $4 \mathrm{~b}$ shows the magnetic field distribution in and around the stack with and without FeCo present. It is worth noting that changing the $n$-value in the model with fixed $h_{1}, w_{1}=92 \mathrm{~mm}$ in the range $n=10-30$ modified the absolute trapped field (higher $n$ value leading to larger field), but the improvement with respect to case with no FM component at $30 \mathrm{~K}$ was maintained at $15-18 \%$.

a)

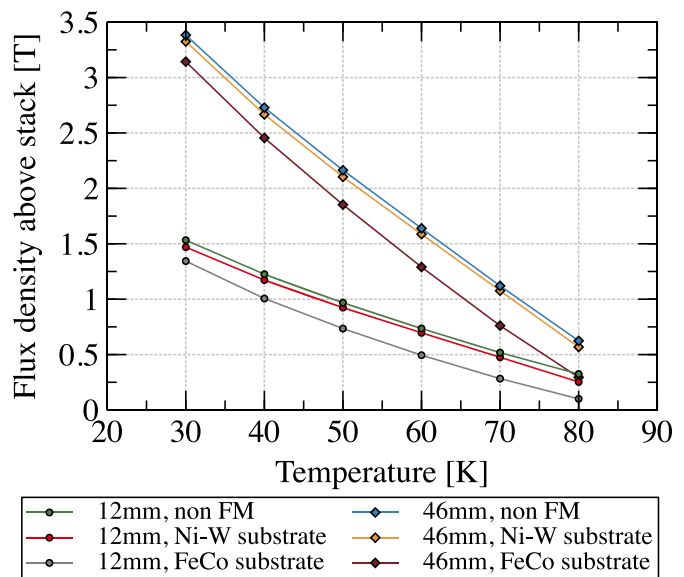

\subsection{Ferromagnetic Substrate and Layered Stack}

Unlike in the case for external ferromagnetic material, it was found that internal ferromagnetic material tends to decrease the flux density outside the material as more flux density is contained within the composite stack itself. Effect is similar for both the layered composite (see Fig. 6b) and a stack where the tape itself is ferromagnetic (homogenised stack with magnetic properties and carrying engineering critical current, akin to the case of ferromagnetic substrate), as shown in Fig. 6a. The flux density outside the stack also decreases with increasing saturation magnetisation of the ferromagnetic material used. Both the current density and magnetic flux density are shown in Fig. 7 for cases 1 and 3 (see Fig. 2a), showing the increased flux density within the stack but not outside it, where it is useful for applications. A lot of detailed theoretical work on magnetisation of thin strips and layered

b)
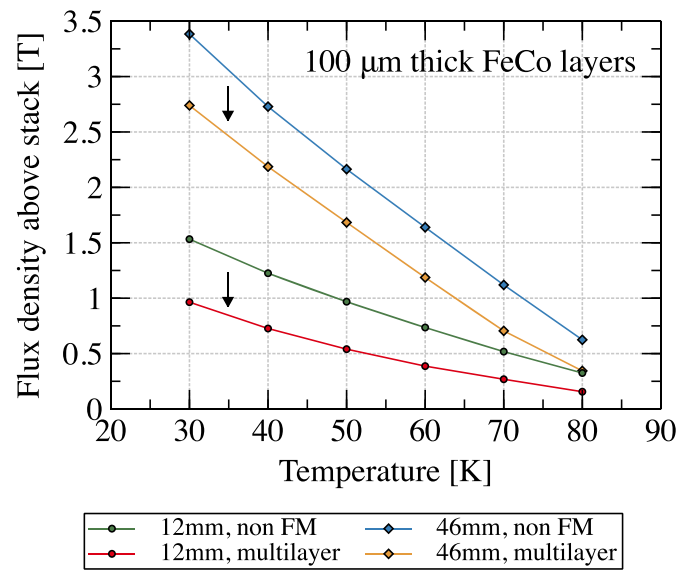

Fig. 6 Flux density above the stacks with and without magnetic properties corresponding to Ni-W and FeCo alloys for 12- and 46-mm stacks (a) and similarly a field above a 60/60 alternating stack of HTS tape and FeCo layers, each $100 \mu \mathrm{m}$ thick (b) 
Fig. 7 Magnetic flux density and current density comparison in an unaltered stack and one that has magnetic properties of FeCo alloy Vacoflux 50, similar to the case of ferromagnetic substrate
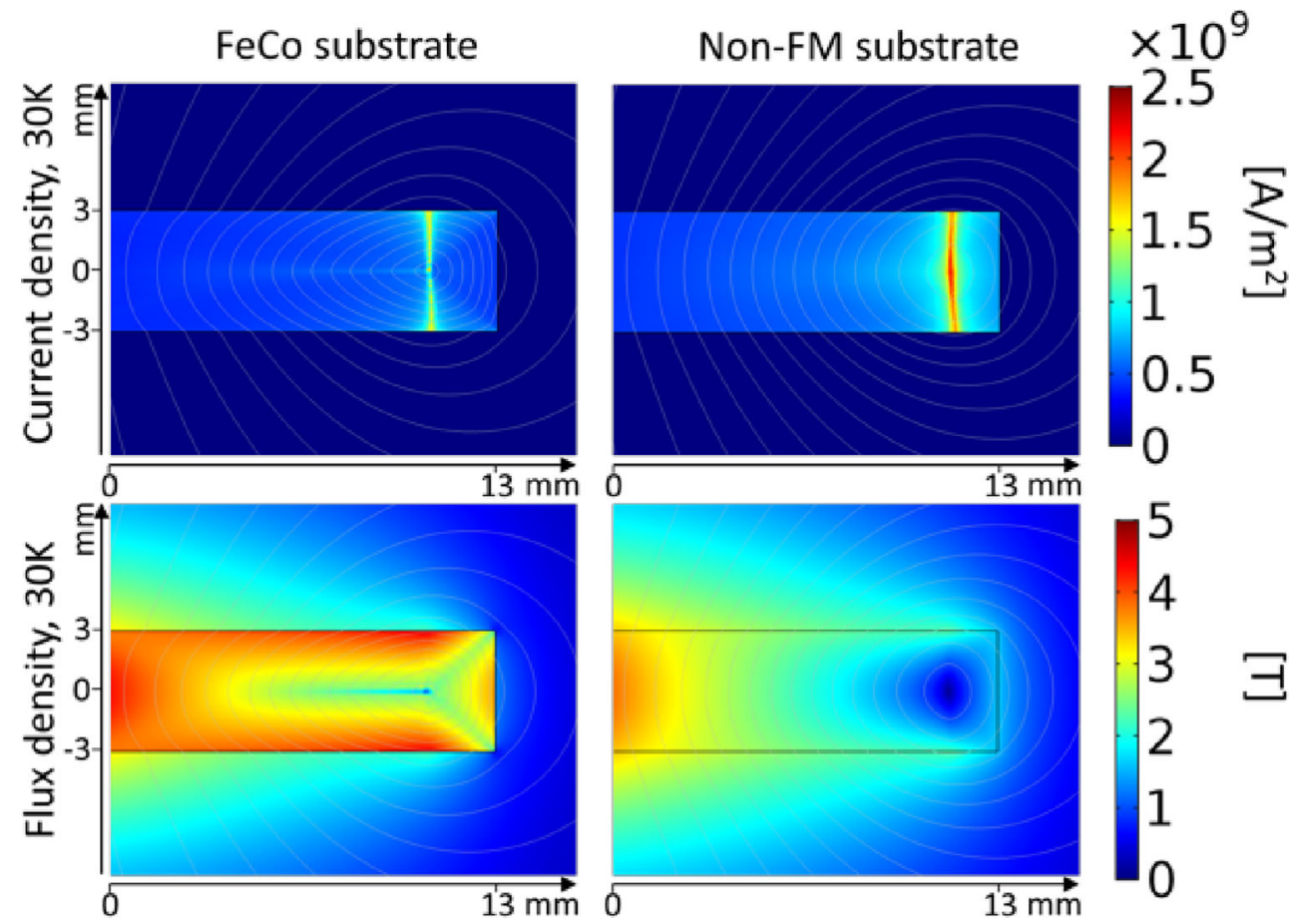

superconductors was done by Brandt [9, 10], where a more rigorous time-dependant treatment with comparison of different $E-J$ laws can be found.

\section{Conclusions}

The results have shown that a substantial enhancement of field above a trapped field magnet is possible by the use of external ferromagnetic material even at flux densities well above the saturation magnetisation of the ferromagnetic material in question. However, internally added ferromagnetic material by layering or otherwise was detrimental to flux density outside the TFM, and the reduction of flux density was worse with ferromagnetic materials with higher saturation magnetisation.

Lastly, it was determined that it is important to consider the anisotropy of critical current density as it changes the flux density profile and the total amount of magnetic flux above the TFM, although it did not change the maximum flux density above the centre of the stack.

Further work is needed to determine the effect of $n$-value dependence on temperature and magnetic field and timedependant simulations should shed light on how flux creep is affected by presence of FM. Additional optimisation, especially moving beyond the simple geometry of the FM and tailored for specific $J_{\mathrm{c}}(B, T, \theta)$ of the superconductor, could lead to higher trapped fields.
Funding Information Algirdas Baskys, Anup Patel and Bartek Glowacki acknowledge the support of an EPSRC grant EP/P000738/1, while Vicente Climente-Alarcon is funded by Horizon 2020 grant 723199.

Open Access This article is distributed under the terms of the Creative Commons Attribution 4.0 International License (http:// creativecommons.org/licenses/by/4.0/), which permits unrestricted use, distribution, and reproduction in any medium, provided you give appropriate credit to the original author(s) and the source, provide a link to the Creative Commons license, and indicate if changes were made.

Publisher's Note Springer Nature remains neutral with regard to jurisdictional claims in published maps and institutional affiliations.

\section{References}

1. Philippe, M.P., Ainslie, M.D., Wéra, L., Fagnard, J.-F., Dennis a, R., Shi, Y.-H., Cardwell, D.A., Vanderheyden, B., Vanderbemden, P.: Influence of soft ferromagnetic sections on the magnetic flux density profile of a large grain, bulk $\mathrm{Y}-\mathrm{Ba}-\mathrm{Cu}-\mathrm{O}$ superconductor. Supercond Sci Technol. 28, 095008 (2015)

2. Philippe, M.P., Fagnard, J.F., Wéra, L., Morita, M., Nariki, S., Teshima, H., Caps, H., Vanderheyden, B., Vanderbemden, P.: Influence of crossed fields in structures combining large grain, bulk (RE)BCO superconductors and soft ferromagnetic discs. J Phys Conf Ser. 695, 012003 (2016)

3. Ainslie, M.D., Fujishiro, H., Mochizuki, H., Takahashi, K., Shi, Y.H., Namburi, D.K., Zou, J., Zhou, D., Dennis, A.R., Cardwell, D.A.: Enhanced trapped field performance of bulk hightemperature superconductors using split coil, pulsed field magnetization with an iron yoke. Supercond Sci Technol. 29, 074003 (2016)

4. Glowacki, B.A., Majoros, M.: Superconducting-magnetic heterostructures: a method of decreasing AC losses and improving 
critical current density in multifilamentary conductors. J Phys Condens Matter. 21, 254206 (2009)

5. Patel, A., Hopkins, S.C., Glowacki, B.A.: Trapped fields up to $2 \mathrm{~T}$ in a $12 \mathrm{~mm}$ square stack of commercial superconducting tape using pulsed field magnetization. Supercond Sci Technol. 26, 032001 (2013)

6. Baskys, A., Patel, A., Hopkins, S., Glowacki, B.: Modeling of trapped fields by stacked (RE)BCO tape using angular transversal field dependency. IEEE Trans Appl Supercond. 26, 6601004 (2016)
7. Wimbush, S.C., Strickland, N.M.: A public database of hightemperature superconductor critical current data. IEEE Trans Appl Supercond. 27, 8000105 (2017)

8. Claassen, J.H., Thieme, C.L.H.: Magnetic properties of Ni-based substrates for HTS tape. Supercond Sci Technol. 21, 105003 (2008)

9. Brandt, E.H.: Superconductors of finite thickness in a perpendicular magnetic field: strips and slabs. Phys Rev B. 54, 4246-4264 (1996)

10. Brandt, E.H.: Maximum pinning energy of vortices in anisotropic and layered superconductors. Europhys Lett. 18, 635-640 (1992) 